\title{
Target Conditioning for One-to-Many Generation
}

\author{
Marie-Anne Lachaux \\ Facebook AI Research \\ malachaux@fb.com
}

\author{
Armand Joulin \\ Facebook AI Research \\ ajoulinefb.com
}

\author{
Guillaume Lample \\ Facebook AI Research \\ glampledfb.com
}

\begin{abstract}
Neural Machine Translation (NMT) models often lack diversity in their generated translations, even when paired with search algorithm, like beam search. A challenge is that the diversity in translations are caused by the variability in the target language, and cannot be inferred from the source sentence alone. In this paper, we propose to explicitly model this oneto-many mapping by conditioning the decoder of a NMT model on a latent variable that represents the domain of target sentences. The domain is a discrete variable generated by a target encoder that is jointly trained with the NMT model. The predicted domain of target sentences are given as input to the decoder during training. At inference, we can generate diverse translations by decoding with different domains. Unlike our strongest baseline (Shen et al., 2019), our method can scale to any number of domains without affecting the performance or the training time. We assess the quality and diversity of translations generated by our model with several metrics, on three different datasets.
\end{abstract}

\section{Introduction}

Neural Machine Translation (NMT) models are trained to translate a sentence from a source language into a target language. There are many translations of the same sentence that are both grammatically correct and faithful to the source, but these translations may differ greatly in their vocabulary, style or grammar. Inferring the best translation among them requires to explore a vast output space to cover this variability. This is typically handle as a post-processing step using a search algorithm, like beam search. This procedure is known to produce translations that lack in diversity, often differing only by a punctuation or a word (Kumar and Byrne, 2004; Li et al., 2016). While the search algorithm can certainly be improved, part of the problem resides also in the training of the NMT models; they are trained on 1-to-1 translation datasets without any objective to encourage diverse translations.

There are many ways to model the diversity of translations from data that contain only one translation, such as mixture of experts (Shen et al., 2019) or variational autoencoders (Zhang et al., 2016). A particularity of machine translation is that it is a one-to-many mapping problem. This means that the variability should be encoded by the target sentence and the question is how to combine a NMT system with a target sentence encoder with no posterior collapse.

In this work, we propose to combine the encoder of the NMT with a discrete target encoder. Similar to other discrete autoencoders (Kaiser et al., 2018; van den Oord et al., 2017), each target sentence is assigned to a discrete variable, or domain, and each domain is associated with an embedding. The embeddings from both encoders are then fed to the decoder of the NMT to form a translation. The discrete latent representation follows a categorical distribution that is constrained to be uniform over the dataset to avoid a mode collapse. Since each domain has its own embedding, changing the domain embedding changes the translation. At test time, we can thus condition the generation on each domain embedding to produce multiple translations with high diversity.

Our approach is general and can be applied on top of any model with little computational overhead. An advantage of our approach is that the number of domains can be arbitrarily large without affecting the performance or the running time. Our approach can replace or work with beam search during inference. We assess the quality and diversity of translations generated by our model with several metrics, on three different datasets. 


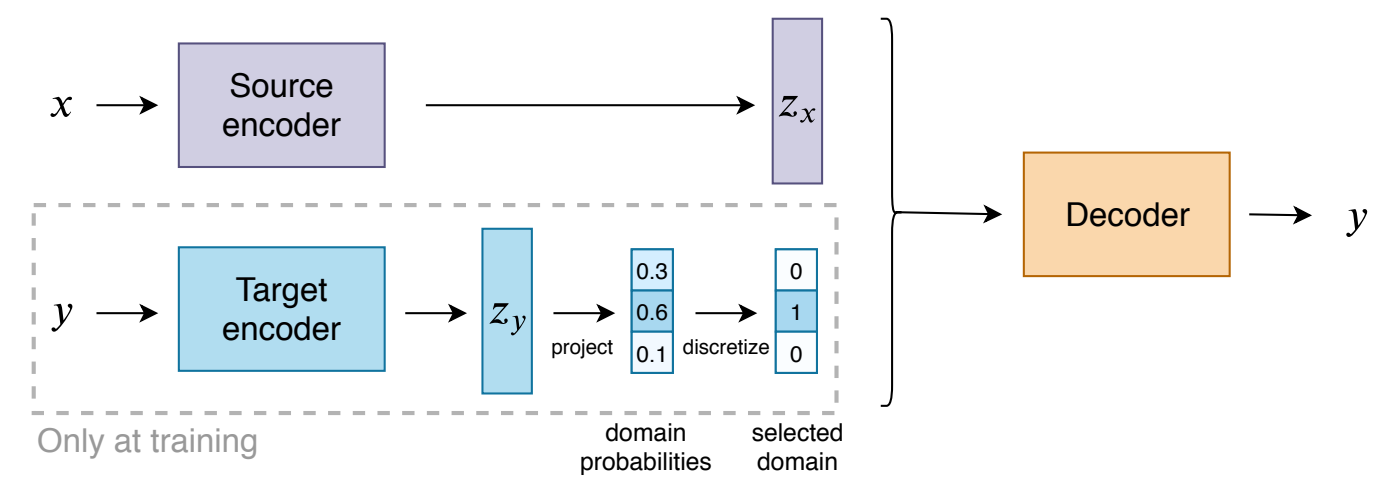

Figure 1: Illustration of our model. The model is composed of a source and a target encoder, and a decoder. At training time, a target sentence is encoded with the target transformer encoder to get a latent representation $z_{y}$. The latent representation is linearly mapped to a vector of size $K$ on which apply a softmax to obtain domain probabilities. Each domain is associated with an embedding. The decoder is fed with both the source encoding, and the sum of the domain embeddings reweighted by their probabilities. During inference, we can generate $K$ different hypotheses by switching the domain embedding that is fed to the decoder. To prevent a train-test discrepancy, during training we apply an argmax operator on domain probabilities, with probability $p_{\text {hard }}$.

\section{Related Work}

Several studies have proposed to sample diverse sequences by changing the value of a latent variable. For example, one possibility is to add noise to the latent space of a Variational AutoEncoder (Kingma and Welling, 2013) to diversify samples in machine translation (Zhang et al., 2016), language modeling (Bowman et al., 2015) or question generation (Jain et al., 2017). In particular, Zhang et al. (2016) also condition the decoder of a NMT Model on a target encoder. As opposed to our work, the output of their encoder is continuous and sampling diverse generation requires to inject random noise, while we obtain diversity by switching between discrete domains. Similar noise injection mechanisms have been investigated to improve the diversity of responses in dialogue (Serban et al., 2017; Cao and Clark, 2017; Wen et al., 2017), and image captioning (Wang et al., 2017; Dai et al., 2017). Closer to our work, (Shen et al., 2019; Shu et al., 2019) and Xu et al. (2018) use domain embeddings to condition their generations. Unlike us, they do not condition the domain on the target, but select the domain which minimizes the reconstruction loss, which becomes expensive as the number of domains increases. Another relevant work is the fast decoder of Kaiser et al. (2018) where they also combine a discrete encoder applied on the target sentence with the NMT encoder. Their goal is to accelerate the decoding process of a machine translation system, while we are interested in efficiently sampling diverse translations.

Another line of work focuses on improving the generation by changing the decoding scheme during inference (Li et al., 2016; Gu et al., 2017) or by matching the training of the model to the decoding scheme (Wiseman and Rush, 2016; Collobert et al., 2019). This is done by either training through a beam search decoder (Wiseman and Rush, 2016; Collobert et al., 2019) or by reframing generation as a reinforcement learning problem (Bengio et al., 2015; Ranzato et al., 2015). These works focus on the decoding scheme to improve generation, but do not address the problem of diversifying the outputs generated from the same input.

\section{Model}

In this section, we describe our target encoder and how to train it along with a translation model. The target encoder learns to map target sentences to discrete domains, and we show how to use these domains to efficiently sample diverse translations.

\subsection{Target encoding}

A Neural Machine Translation (NMT) model is composed of a source encoder $E_{\mathrm{src}}$, and a decoder $D$. Given a dataset $\mathcal{D}$ of pairs $(x, y)$ of source sentences and their target translations, a standard encoder-decoder model is trained to minimize:

$$
\mathbb{E}_{(x, y) \in \mathcal{D}}\left(-\log p_{D}\left(y \mid E_{\text {src }}(x)\right)\right)
$$

where $p_{D}\left(y \mid E_{\mathrm{src}}(x)\right)$ represents the probability given by the decoder $D$ to a target sentence $y$ to be the translation of a source sentence $x$. In our case, we consider that we also have a target encoder $E_{\mathrm{tgt}}$, 
and we feed the decoder not only with an encoding of the source sentence, but also with an encoding of the target sentence. As a result, the model is trained to minimize:

$$
\mathbb{E}_{(x, y) \in \mathcal{D}}\left(-\log p_{D}\left(y \mid E_{\mathrm{src}}(x) ; E_{\mathrm{tgt}}(y)\right)\right)
$$

Without architectural constraint, the decoder $D$ could trivially learn the identity mapping between the encoding of the target sentence $E_{\text {tgt }}(y)$ and the sentence to generate $y$. Instead, we propose to use a key-value structure for this embedding where the target encoder provides a probability for a key, or domain, and we feed the associated value to the decoder of the machine translation system. In practice, we constraint the output of the target encoder to represent the domain probability distribution of the target sentence. The output of the target encoder is thus a $K$-dimensional vector of probabilities $p=E_{\mathrm{tgt}}(y)$. Since the output of the target encoder is not directly fed to the decoder, we bound the amount of information provided by the target encoder, preventing the model from learning a trivial mapping. At test time, we cannot estimate $E_{\text {tgt }}(y)$ since the target sentence $y$ is not available. Instead, we feed the decoder $D$ with any one-hot vector of $\mathbb{R}^{K}$ to generate $K$ different translations. An illustration of our model is provided in Figure 1.

\subsection{Implementation}

Our NMT model is the transformer network of Vaswani et al. (2017) with a dimension $d$, with a transformer encoder $E_{\text {src }}$ and a transformer decoder $D$. The target encoder $E_{\text {tgt }}$ that we introduce in this paper is composed of a transformer encoder with the same architecture as the source encoder $E_{\text {src }}$ and other components detailed bellow. We refer the reader to Vaswani et al. (2017) for the details of the architecture and describe below the specificites of our target encoder $E_{\text {tgt }}$.

The output $E_{\mathrm{tgt}}(y)$ of the target encoder is a probability vector of size $K$. To obtain these probabilities, we encode the target through a transformer encoder. We take the first hidden state $h \in \mathbb{R}^{d}$ of the last layer of the target encoder, corresponding to the start token. We linearly map $h$ to a score vector of dimension $K$. Finally, we apply a softmax operator to obtain a vector of domain probabilities:

$$
p=E_{\text {tgt }}(y)=\operatorname{softmax}(M h)
$$

In that setting, the decoder is trained with arbitrary probability vectors, which becomes problematic at test time when $p$ is set to a one-hot embedding on which the decoder may never have been trained. To prevent this train-test discrepancy, we apply a temperature on the domain scores $s$ that decreases linearly from 1 to 0 over training. When the temperature reaches 0 , we have $p=\mathcal{I}(\operatorname{argmax}(s))^{1}$ (i.e. the domain with the highest score has probability 1 , the others have probability 0 ) and the target encoder remains frozen during the remaining training time.

Moreover, at each training step, we randomly replace the softmax by an argmax operator with a probability $p_{\text {hard }}$. In practice, we set $p_{\text {hard }}=0.25$, which means that $75 \%$ of the time the target encoder is trained along with the source encoder and decoder, and $25 \%$ of the time the target encoder is only used to predict the domain with the highest probability. Overall, we have:

$$
E_{\text {tgt }}(y)= \begin{cases}\mathcal{I}(\operatorname{argmax}(s)), & \text { if } 0 \leq X \leq p_{\text {hard }} \\ \operatorname{softmax}\left(\frac{s}{T}\right), & \text { otherwise }\end{cases}
$$

where $X$ is a random variable from a uniform distribution, i.e., $X \sim \mathcal{U}(0,1)$.

Optimization. When $T>0$, the model is fully differentiable and the target encoder can be trained in an end-to-end fashion with the rest of the model. We found that it is also possible to use discrete operators like the Gumbel-Softmax (Jang et al., 2016). This way, $E_{\text {tgt }}(y)$ is always a one-hot vector and there is no train-test discrepancy. However, learning the target encoder through a discrete encoding makes optimization more difficult, and we obtained better results with a regular softmax.

Domain input. To feed the target encoder output $E_{\mathrm{tgt}}(y)$ as input to the decoder $D$, the decoder learns a matrix of embeddings $E=\left[e_{0}, \ldots, e_{K-1}\right] \in \mathbb{R}^{d \times K}$ where each $e_{i}$ represents a different domain. Traditionally, the first input of a decoder is an embedding that corresponds to a start symbol $\langle S\rangle$. Instead, we feed as first embedding a vector $e$, where:

$$
e=E p=\sum_{i=0}^{K-1} p_{i} e_{i}, \quad \text { with } \quad p=E_{\text {tgt }}(y)
$$

\footnotetext{
${ }^{1} \operatorname{By} \mathcal{I}(j)=(0, \ldots, 0,1,0, \ldots, 0)$, we denote the one-hot vector with 1 for $j$-th coordinate and 0 elsewhere).
} 
The domain embeddings $E$ are learned during training. This process is illustrated in Figure 2.

\subsection{Training objective}

We denote by $\theta$ the parameters of $E_{\mathrm{src}}, E_{\mathrm{tgt}}$, and $D$. Given a mini-batch of source and target sentences $\left\{\left(x_{i}, y_{i}\right)\right\}_{1 \leq i \leq N}$, the model is trained to minimize:

$$
\mathcal{L}(\theta)=\sum_{i=1}^{N}-\log \left(p_{D}\left(y_{i} \mid E_{\mathrm{src}}\left(x_{i}\right) ; E_{\mathrm{tgt}}\left(y_{i}\right)\right)\right)
$$

In practice, we want the decoder to properly leverage $E_{\text {tgt }}(y)$, i.e., the domain information coming from the target encoder. Without additional constraints, nothing prevents the model from collapsing to a mode where the target encoder constantly predicts the same domain, regardless of its input. The model is then perfectly predicting its domain, which means that it receives no gradient to escape this trivial solution.

To address this issue, we add a regularization term to the training objective, to encourage the model to make a uniform usage of available domains. In particular, we define the entropy distribution of selected domains in the mini-batch:

$$
\mathcal{L}_{X E}(\theta)=-\widetilde{p} \log (\widetilde{p}), \quad \text { with } \quad \widetilde{p}=\frac{1}{N} \sum_{i=1}^{N} p_{i}
$$

where $p_{i}=E_{\text {tgt }}\left(y_{i}\right)$ is the probability distribution of domains for the target sentence $y_{i}$. Finally, the model is trained to minimize $\mathcal{L}(\theta)-\lambda \mathcal{L}_{X E}(\theta)$, where $\lambda$ is a hyper-parameter.

\subsection{Inference}

At inference, we generate one hypothesis per domain, i.e. $K$ hypotheses. To generate the $k^{\text {th }}$ hypothesis, we perform decoding by feeding $e_{k}$ as embedding of the start symbol. We generate translations with greedy decoding, except in Figure 5, where we combine our model with beam search decoding which leads to a different quality vs. diversity trade-off.

\section{Experiments}

In this section, we describe an evaluation protocol similar to Shen et al. (2019), and compare our approach to several baselines on 3 MT datasets. Then, we show the importance of different components in our model in an ablation study.

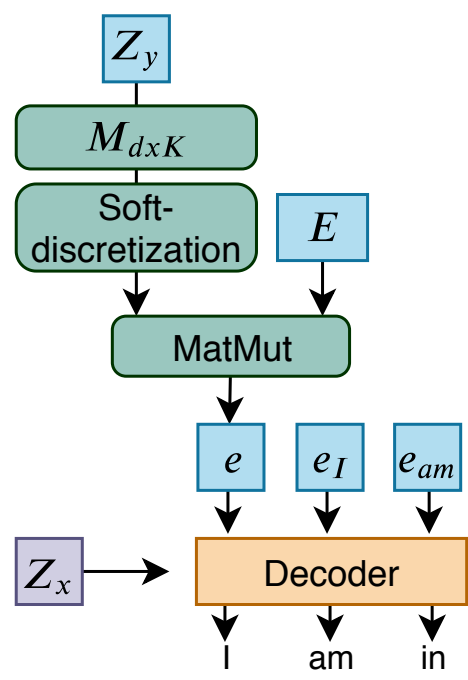

Figure 2: Detailed illustration of our model. $Z_{y}$ is the first hidden state of the output of the target transformer encoder. To obtain $E_{\text {tgt }}(y)$, we linearly map $Z_{y}$ to a $K$ dimensional vector and perform a "soft-disctretization" by applying either a softmax or an argmax operator. We then compute the target domain vector $e$ as the sum of the domain embeddings $E$ reweighted by their probabilities contained in $E_{\text {tgt }}(y)$. The vector $e$ is fed to the decoder as the embedding of the first token, along with the source encoding $Z_{x}=E_{\mathrm{src}}(x)$.

\subsection{Evaluation Metrics}

To measure both the quality and diversity of our generations, we use an evaluation protocol similar to Shen et al. (2019). The test set has multiple human reference translations which allows to measure diversity. Formally, we denote by $\left\{s_{i},\left[r_{i}^{1}, \ldots, r_{i}^{P}\right]\right\}_{1 \leq i \leq N}$ a multi-reference dataset, where each source sentence $s_{i}$ is provided with $P$ reference translations $\left[r_{i}^{1}, \ldots, r_{i}^{P}\right]$, and by $\left[h_{i}^{1}, \ldots, h_{i}^{K}\right]$ the $K$ hypotheses generated by our model for the source sentence $s_{i}$.

We denote by $\operatorname{BLEU}\left(\left\{h_{i},\left[r_{i}^{1}, \ldots, r_{i}^{P}\right]\right\}_{1<i<N}\right)$ the corpus-level BLEU score, with $P \geq 1$ references for each hypothesis. To measure the quality of our generations, we define:

$$
\operatorname{mBLEU}=\operatorname{BLEU}\left(\left\{h_{i}^{j},\left[r_{i}^{1}, \ldots, r_{i}^{P}\right]\right\}_{1 \leq i \leq N, j \in K}\right)
$$

mBLEU measures the quality of translations for each source sentence, and for each domain. A model that does not generate good translations for each domain will perform poorly. To measure the diversity of translations, we use the pairwise metric of Shen et al. (2019), defined as:

pairwise $=\operatorname{BLEU}\left(\left\{h_{i, j},\left[h_{i, k}\right]\right\} \underset{\substack{1 \leq i \leq N \\(j, k) \in K^{2}, j \neq k}}{ }\right)$ 
pairwise computes the BLEU score between hypotheses of a same source sentence. A low pairwise ensures diversity in translations, while a pairwise of 100 means that for a given source sentence, the decoder will always generate the same translation. Overall, we want the model to have a low pairwise while preserving a high $\mathrm{mBLEU}$ score.

\subsection{Dataset}

We train and test our model on three different datasets, following Shen et al. (2019). Each dataset comes with a test set with multiple human reference translations.

WMT'17 English-German (En-De). We follow the same pre-processing protocol as Shen et al. (2019), where we filter all training sentences with more than 80 source or target words, which results in 4.5M sentence pairs. We apply the Moses tokenizer (Koehn et al., 2007) and learn a joint BPE vocabulary with $32 \mathrm{k}$ codes (Koehn et al., 2007). We take newstest2013 as a validation set, and test on a subset of 500 sentences of newstest 2014 with 10 reference translations.

WMT'14 English-French (En-Fr). We follow the setup of Gehring et al. (2017), which results in $36 \mathrm{M}$ training sentence pairs. We use a joint vocabulary of 40k BPE codes. We use newstest 2012 and newstest 2013 as a validation set, and test on a subset of 500 sentences from newstest 2014 with 10 reference translations.

WMT'17 Chinese-English (Zh-En). We follow the pre-processing setup of Hassan et al. (2018). The training set is composed of 20M sentence pairs, with $48 \mathrm{k}$ and $32 \mathrm{k}$ source and target BPE vocabularies respectively. We develop on devtest2017 and evaluate on a subset of 2000 sentences of newstest 2017 that comes with 3 reference translations.

\subsection{Experimental details}

In all our experiments, we consider transformers with 6 layers, 8 attention heads, and we set the model dimension to $d=512$. We optimize our model with the Adam optimizer (Kingma and $\mathrm{Ba}$, 2014) with $\beta_{1}=0.9, \beta_{2}=0.98$ and a learning rate of $3 \times 10^{-4}$. We use the same learning rate schedule as Vaswani et al. (2017). We use a dropout (Srivastava et al., 2014) of 0.1 in the source encoder and the decoder. Following Shen et al. (2019), we do not use any dropout in the target encoder. With stochasticity in the target encoder, a same target sentence tends to be mapped to different domains at different iterations, which prevents the decoder from learning the specificity of each domain, and results in identical generations with no diversity.

We use 128 GPUs for the En-Fr experiments, and 16 GPUs for the En-De and Zh-En experiments. For the En-Fr experiments, we train with minibatches of around $450 \mathrm{k}$ tokens, and $55 \mathrm{k}$ tokens for En-De and Zh-En. We use float 16 operations to speed up training and to reduce the memory usage of our models. We implement our model within the fairseq framework of Ott et al. (2019).

\subsection{Baselines}

Sampling and Beam. We report results with a sampling and a beam baseline, as well as the diverse beam method (Vijayakumar et al., 2018). We consider a standard NMT system (i.e. an encoderdecoder model, without target encoder or latent variable). At test time, for sampling we sample $K$ translations to generate $K$ hypotheses. For the beam search, we use a beam size of $K$ and return all hypotheses in the beam.

Mixture of Experts. We also compare against the state-of-the-art Mixture of Experts (MoE) model of Shen et al. (2019), with online responsibility update, uniform prior, shared parameters and hard assignment ( $h M u p$ in their paper), which is their overall best setup. MoE model is composed of a source encoder $E_{\mathrm{src}}$ and a decoder $D$. Like our model, the decoder learns a matrix of embeddings $E=\left[e_{0}, \ldots, e_{K-1}\right] \in \mathbb{R}^{d \times K}$ where each $e_{i}$ represents a different domain which is fed as first input of the decoder. Unlike us, they do not use a separate target encoder to select the domain, but consider an EM algorithm where the selected domain is the one that minimizes the reconstruction loss of the target sentence. In particular, for a mini-batch of $N$ source and target sentences $\left\{\left(x_{i}, y_{i}\right)\right\}_{1 \leq i \leq N}$, the E-step computes:

$$
d_{i}^{*}=\underset{d \in[1, K]}{\operatorname{argmax}} p_{D}\left(y_{i} \mid E_{\mathrm{src}}\left(x_{i}\right) ; d\right)
$$

Then, the M-step minimizes the negative logprobability of target sentences, given their source encodings, and the selected domains:

$$
\mathcal{L}(\theta)=\sum_{i=1}^{N}-\log \left(p_{D}\left(y_{i} \mid E_{\mathrm{src}}\left(x_{i}\right) ; d_{i}^{*}\right)\right)
$$




\begin{tabular}{lcccccccc}
\hline & \multicolumn{3}{c}{ mBLEU } & & \multicolumn{3}{c}{ pairwise } \\
\cline { 2 - 3 } & En-De & En-Fr & Zh-En & & En-De & En-Fr & Zh-En \\
\hline Sampling & 28.2 & 43.6 & 19.1 & & 11.8 & 21.0 & 12.0 \\
Beam & 66.3 & 79.3 & 32.2 & & 74.0 & 77.7 & 83.8 \\
Diverse Beam (Vijayakumar et al., 2018) & 60.0 & 72.5 & 31.6 & & 53.7 & 64.9 & 66.5 \\
MoE (Shen et al., 2019) & 59.8 & 72.6 & 35.7 & & 48.8 & 64.4 & 47.5 \\
Our model & 55.4 & 65.9 & 34.7 & & 46.2 & 57.3 & 52.5 \\
\hline
\end{tabular}

Table 1: Results on three WMT datasets: En-De, En-Fr, Zh-En. We use $K=10,10,3$ domains respectively. We generate the same number of hypotheses as the number of references available in the multi-references datasets. Beam search is computed with beam size of $K$.

We run all of these baselines with the same transformer architecture as the one used in our model. For fair comparison, we use the same optimizer, learning rate and batch size in all experiments.

\subsection{Main results}

Table 1 present mBLEU and pairwise scores for different models, on the three considered datasets. We observe that a high mBLEU score is often combined with a high pairwise. For instance, the beam search and sampling baselines fail at generating both diverse and high quality translations. Beam search and diverse beam search hypotheses are accurate, but lack diversity, resulting in a very similar set of hypotheses. On WMT En-De, with $K=10$, beam search gives a $\mathrm{MBLEU}$ score of 66.3 but a pairwise score of 74 . On the other hand, the sampling baseline generates very diverse but inaccurate hypotheses, with a pairwise score of 11.8, but a MBLEU of 28.2.

The Mixture of Experts and Target Encoder models have a better trade-off between diversity and quality, as shown in Figure 4. Overall, our method provides more diversity than the MoE method, i.e. it obtains a lower pairwise score, but to the detriment of a lower mBLEU score. In Table 1, we observe that for En-De and En-Fr, our model obtains a lower MBLEU score than beam search decoding and the Mixture of Experts, but provides more diversity, with a pairwise score of 57.3 instead of 64.4 in En-Fr. While both methods perform similarly, our approach is simpler to implement, and can easily scale to an arbitrary number of domains, as shown in the following section.

\subsection{Training speed}

The training speed of our method is independent of the number of domains. In contrast, the train-

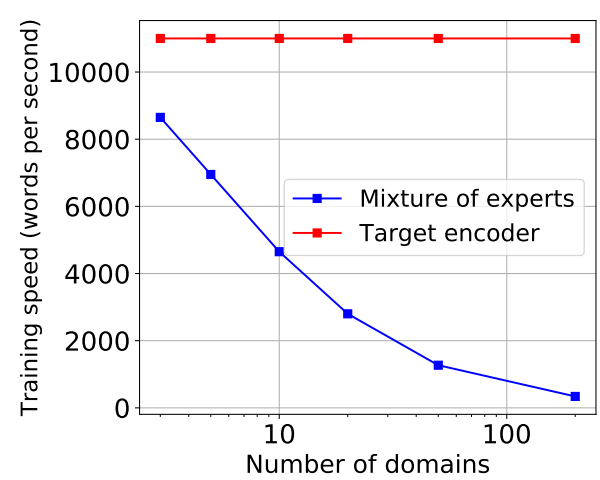

Figure 3: Training speed. Measured in number of words per second, for our target encoder model and the Mixture of Experts model of Shen et al. (2019), for different number of domains ( $K=3,5,10,20,50,200)$. The training speed of the target encoder model is constant while the Mixture of Experts model training speed decreases with the number of domains.

ing speed of the MoE model of Shen et al. (2019) decreases drastically when the number of domains increases. Indeed, the MoE model requires to perform $K$ forward passes to determine the best domain. In Figure 3, we compare the training speed of both models for $K=3,5,10,20,50$ and 200. Unlike the MoE model, using a target encoder allows generalization to an arbitrary number of domains.

\subsection{Ablation study}

Beam search. In Figure 5, we study the impact of decoding with beam search instead of greedy decoding. Using beam search improves the quality of translations, but deteriorates the diversity. Combining a target encoder model with a beam search pushes towards the same trade-off of qualitydiversity as the greedy MoE model.

Domain regularization. Without any regularization on the domain probabilities, i.e. when $\lambda=0$, 


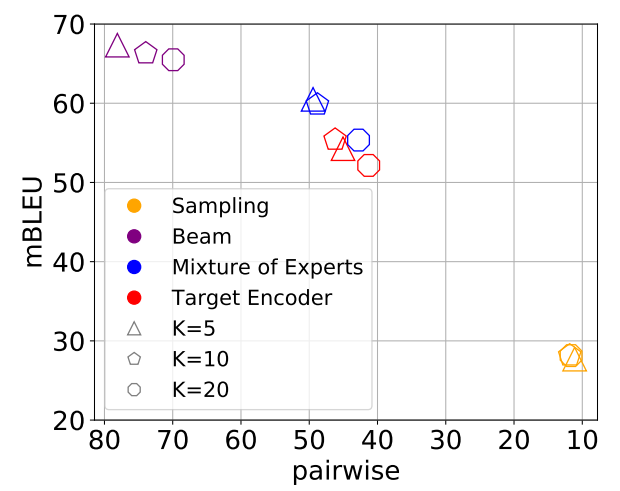

Figure 4: Impact of the number of domains. Results on the WMT'17 En-De dataset. We compare beam search, sampling, MoE (Shen et al., 2019) to our Target Encoder. In each case, we report results for $K=5,10$ and 20 domains. MoE and Target Encoder provide the best trade-off between quality and diversity. Compared to MoE, Target Encoder provides a lower MBLEU score, but also a lower pairwise (i.e., more diversity).

we sometimes encounter the "collapse" scenario where at training time all target sentences are mapped to the same domain. As a result, only the embedding associated to that domain is trained, and at test time, every sentence generated from another (and untrained) domain embedding will be invalid. This means that only one of the $K$ generated hypotheses will be valid, leading to a very poor mBLEU. Conversely, when $\lambda$ is too high, the regularization term becomes predominant and the target encoder primarily focuses on maximizing the domain usage entropy, rather than on minimizing the decoder reconstruction loss. As a result, the target encoder uniformly maps target sentences to all available domains, but the domains do not contain any information about target sentences. This way, the decoder learns to ignore the domain, and will always output the same translation, independently

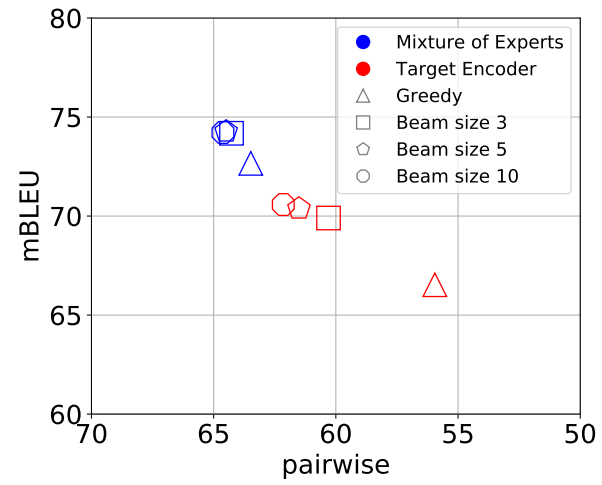

Figure 5: Beaming search decoding. Results on the WMT'14 En-Fr dataset for $K=10$ domains. We study the impact of decoding greedily and beam search, for beam sizes of 3,5 and 10 . Beam search increases both the mBLEU and the pairwise scores, i.e., it provides higher quality translations, but with lower diversity.

of the input domain, which results in a pairwise score close to 100 (i.e. there is no diversity). In practice, we found that setting $\lambda=0.1$ or $\lambda=1$ leads to similar results, and is enough to prevent the collapse scenario.

Source versus target encoding. In this experiment, we change the input of our target encoder to probe where the source of diversity in our model comes from. In particular, it is possible that the diversity captured by our model is indirectly coming from the source sentences through the target sentences. We test this hypothesis by replacing the input of the target encoder by the source sentence. This model is identical to ours beside the change in the input of the target encoder. In that setting, on WMT'17 En-De, when using 10 domains, we obtain a mBLEU score of 66.5, and a pairwise BLEU of 97.2 , which means that the model was not able to learn anything specific about each domain,

\begin{tabular}{|c|c|c|}
\hline Source & 参与投票的成员中, $58 \%$ 反对该合同交易。 & 自11月份开始, 俄罗斯民意也有所扭转。 \\
\hline \multirow[t]{3}{*}{ Human references } & It was rejected by $58 \%$ of its members who voted in the ballot. & Russian public opinion has also turned since November. \\
\hline & Of the members who voted, $58 \%$ opposed the contract transaction. & Russian public opinion has started to change since November. \\
\hline & Of the members who participated in the vote, $58 \%$ opposed the contract. & The polls in Russian show a twist turn since the beginning of November. \\
\hline \multirow[t]{3}{*}{ Beam 3, Top 3} & Of those voting, 58 per cent opposed the contract deal. & Since November, Russian public opinion has also turned around. \\
\hline & Fifty-eight per cent of the members voting opposed the contract deal. & Since November, Russian public opinion has also changed. \\
\hline & Fifty-eight per cent of the members voting opposed the contract. & Russian public opinion has also changed since November. \\
\hline \multirow{3}{*}{$\begin{array}{l}\text { Mixture of Experts } \\
\text { (Shen et al., 2019) }\end{array}$} & Of the members who voted, $58 \%$ opposed the deal. & Since November, the mood in Russia has also reversed. \\
\hline & Fifty-eight per cent of the members who voted opposed the contract deal. & Since November, opinion in Russia has also reversed. \\
\hline & Fifty-eight per cent of the voting members opposed the contract deal. & Opinion in Russia has also shifted since November. \\
\hline \multirow[t]{3}{*}{ Our Model } & Of the members voting, 58 per cent opposed the contract deal. & Since November, Russian public opinion has also reversed. \\
\hline & Fifty-eight per cent of the members who voted opposed the contract deal. & The mood in Russia has also reversed since November. \\
\hline & Fifty-eight per cent of those voting had opposed this contract deal. & There has also been a reversal in Russian public opinion since November. \\
\hline
\end{tabular}

Table 2: Two examples of generations by our model and different baselines on the WMT'17 Zh-En dataset. Beam search generation lack diversity. The target encoder model gives the most diverse sets of translations. 


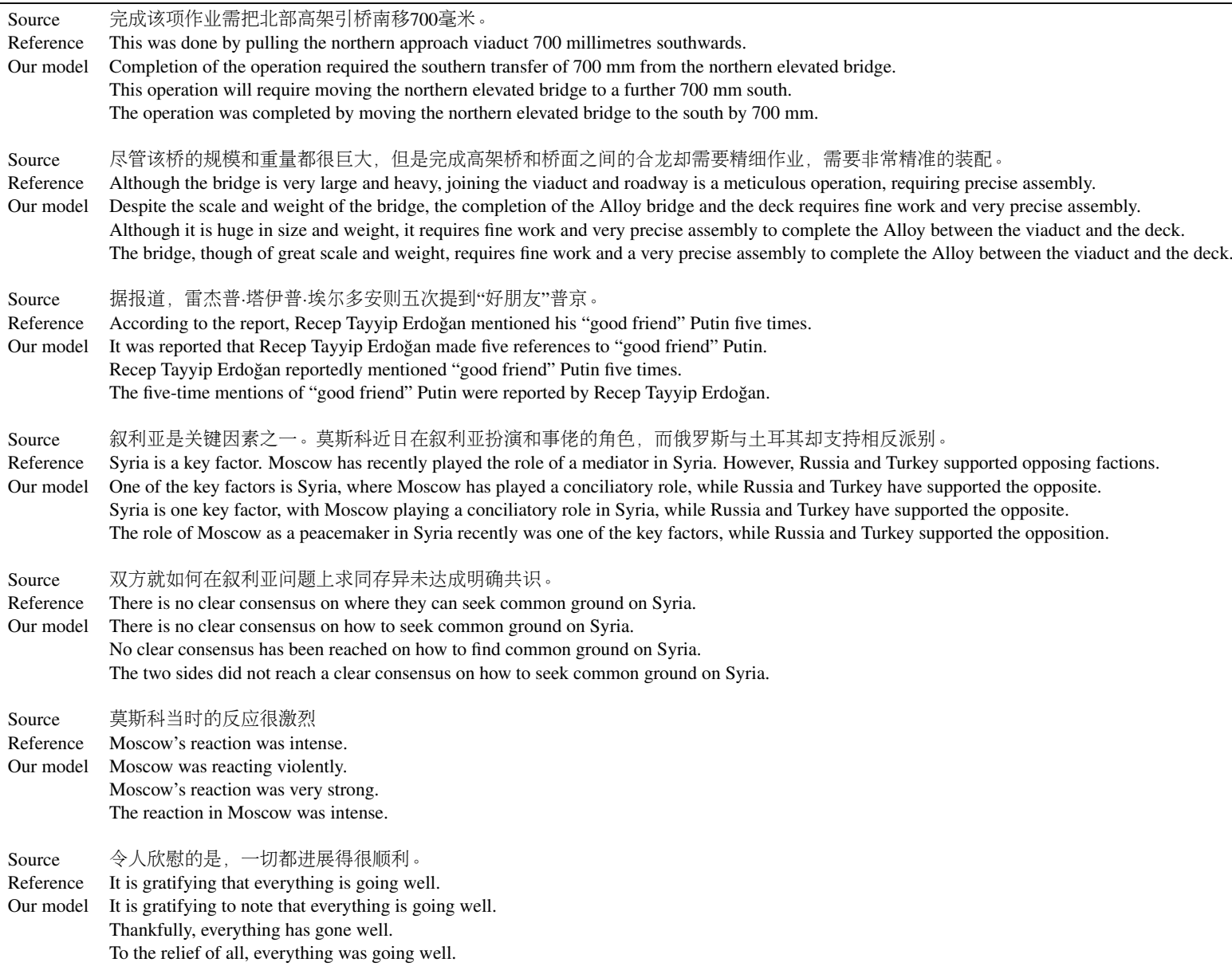

Table 3: Examples of generations by our model on the WMT'17 Zh-En dataset. Translations are sorted by domain index. The model generates high-quality translations with high diversity. Unlike beam search decoding, that tends to return similar hypotheses with only minor differences, our model generates various translations with very different prefixes, even for long sentences.

and the decoder simply ignores the domain information. The fact that learning the domain from the input sentence does not work well is expected, as this information is already encoded in the source encoding $z_{x}$. This validates that learning the diversity form the target domains is important. It also suggests that the diversity that our model learns is inherent to the target domain, and does not come from the source domain indirectly. Finally, both models have the same number of parameters, suggesting that the gain in performance is not only caused by the additional parameters.

\subsection{Qualitative analysis}

Table 3 provides examples of generations by our model on the WMT'17 Zh-En dataset. For each Chinese source sentence, we provide one English human translated reference, and translations by our model for three different domains. We observe that the model generates high-quality translations with high diversity. Unlike beam search decoding, that tends to return similar hypotheses with only minor differences in the suffix (Ott et al., 2018), our model is able to generate diverse translations with very different prefixes, even for long sentences.

\section{Conclusion}

In this paper, we presented an efficient way to sample diverse translations by adding a discrete target encoder to a NMT model. The discrete representation allows to change the domain of the translation and can be trained without supervision. The advantages of using a discrete encoder is that it is both general and scales with the number of domains with no additional computational time. In the future, we plan to test our discrete target encoder to diversify generations in other domains, such as language modeling, image captioning or image inpainting. 


\section{References}

Samy Bengio, Oriol Vinyals, Navdeep Jaitly, and Noam Shazeer. 2015. Scheduled sampling for sequence prediction with recurrent neural networks. In Advances in Neural Information Processing Systems, pages 1171-1179.

Samuel R Bowman, Luke Vilnis, Oriol Vinyals, Andrew M Dai, Rafal Jozefowicz, and Samy Bengio. 2015. Generating sentences from a continuous space. arXiv preprint arXiv:1511.06349.

Kris Cao and Stephen Clark. 2017. Latent variable dialogue models and their diversity. arXiv preprint arXiv: 1702.05962.

Ronan Collobert, Awni Hannun, and Gabriel Synnaeve. 2019. A fully differentiable beam search decoder. arXiv preprint arXiv:1902.06022.

Bo Dai, Sanja Fidler, Raquel Urtasun, and Dahua Lin. 2017. Towards diverse and natural image descriptions via a conditional gan. In Proceedings of the IEEE International Conference on Computer Vision, pages 2970-2979.

Jonas Gehring, Michael Auli, David Grangier, Denis Yarats, and Yann N Dauphin. 2017. Convolutional sequence to sequence learning. In Proceedings of the 34th International Conference on Machine Learning-Volume 70, pages 1243-1252. JMLR. org.

Jiatao Gu, Kyunghyun Cho, and Victor OK Li. 2017. Trainable greedy decoding for neural machine translation. arXiv preprint arXiv:1702.02429.

Hany Hassan, Anthony Aue, Chang Chen, Vishal Chowdhary, Jonathan Clark, Christian Federmann, Xuedong Huang, Marcin Junczys-Dowmunt, William Lewis, Mu Li, et al. 2018. Achieving human parity on automatic chinese to english news translation. arXiv preprint arXiv:1803.05567.

Unnat Jain, Ziyu Zhang, and Alexander G Schwing. 2017. Creativity: Generating diverse questions using variational autoencoders. In Proceedings of the IEEE Conference on Computer Vision and Pattern Recognition, pages 6485-6494.

Eric Jang, Shixiang Gu, and Ben Poole. 2016. Categorical reparameterization with gumbel-softmax. arXiv preprint arXiv:1611.01144.

Łukasz Kaiser, Aurko Roy, Ashish Vaswani, Niki Parmar, Samy Bengio, Jakob Uszkoreit, and Noam Shazeer. 2018. Fast decoding in sequence models using discrete latent variables. arXiv preprint arXiv:1803.03382.

Diederik Kingma and Jimmy Ba. 2014. Adam: A method for stochastic optimization. arXiv preprint arXiv:1412.6980.

Diederik P Kingma and Max Welling. 2013. Autoencoding variational bayes. arXiv preprint arXiv:1312.6114.
Philipp Koehn, Hieu Hoang, Alexandra Birch, Chris Callison-Burch, Marcello Federico, Nicola Bertoldi, Brooke Cowan, Wade Shen, Christine Moran, Richard Zens, et al. 2007. Moses: Open source toolkit for statistical machine translation.

Shankar Kumar and William Byrne. 2004. Minimum bayes-risk decoding for statistical machine translation. Technical report.

Jiwei Li, Will Monroe, and Dan Jurafsky. 2016. A simple, fast diverse decoding algorithm for neural generation. arXiv preprint arXiv:1611.08562.

Aaron van den Oord, Oriol Vinyals, et al. 2017. Neural discrete representation learning. In Advances in Neural Information Processing Systems, pages 6306-6315.

Myle Ott, Michael Auli, David Grangier, and Marc'Aurelio Ranzato. 2018. Analyzing uncertainty in neural machine translation. arXiv preprint arXiv:1803.00047.

Myle Ott, Sergey Edunov, Alexei Baevski, Angela Fan, Sam Gross, Nathan Ng, David Grangier, and Michael Auli. 2019. fairseq: A fast, extensible toolkit for sequence modeling. In Proceedings of NAACL-HLT 2019: Demonstrations.

Marc'Aurelio Ranzato, Sumit Chopra, Michael Auli, and Wojciech Zaremba. 2015. Sequence level training with recurrent neural networks. arXiv preprint arXiv:1511.06732.

Iulian Vlad Serban, Alessandro Sordoni, Ryan Lowe, Laurent Charlin, Joelle Pineau, Aaron Courville, and Yoshua Bengio. 2017. A hierarchical latent variable encoder-decoder model for generating dialogues. In Thirty-First AAAI Conference on Artificial Intelligence.

Tianxiao Shen, Myle Ott, Michael Auli, and Marc'Aurelio Ranzato. 2019. Mixture models for diverse machine translation: Tricks of the trade. arXiv preprint arXiv:1902.07816.

Raphael Shu, Hideki Nakayama, and Kyunghyun Cho. 2019. Generating diverse translations with sentence codes. In Proceedings of the 57th Annual Meeting of the Association for Computational Linguistics, pages 1823-1827.

Nitish Srivastava, Geoffrey Hinton, Alex Krizhevsky, Ilya Sutskever, and Ruslan Salakhutdinov. 2014. Dropout: a simple way to prevent neural networks from overfitting. The journal of machine learning research, 15(1):1929-1958.

Ashish Vaswani, Noam Shazeer, Niki Parmar, Jakob Uszkoreit, Llion Jones, Aidan N Gomez, Łukasz Kaiser, and Illia Polosukhin. 2017. Attention is all you need. In Advances in neural information processing systems, pages 5998-6008. 
Ashwin K Vijayakumar, Michael Cogswell, Ramprasaath R Selvaraju, Qing Sun, Stefan Lee, David Crandall, and Dhruv Batra. 2018. Diverse beam search for improved description of complex scenes. In Thirty-Second AAAI Conference on Artificial Intelligence.

Liwei Wang, Alexander Schwing, and Svetlana Lazebnik. 2017. Diverse and accurate image description using a variational auto-encoder with an additive gaussian encoding space. In Advances in Neural Information Processing Systems, pages 5756-5766.

Tsung-Hsien Wen, Yishu Miao, Phil Blunsom, and Steve Young. 2017. Latent intention dialogue models. In Proceedings of the 34th International Conference on Machine Learning-Volume 70, pages 3732-3741. JMLR. org.

Sam Wiseman and Alexander M Rush. 2016. Sequence-to-sequence learning as beam-search optimization. arXiv preprint arXiv:1606.02960.

Qiongkai Xu, Juyan Zhang, Lizhen Qu, Lexing Xie, and Richard Nock. 2018. D-page: Diverse paraphrase generation. arXiv preprint arXiv:1808.04364.

Biao Zhang, Deyi Xiong, Hong Duan, Min Zhang, et al. 2016. Variational neural machine translation. In Proceedings of the 2016 Conference on Empirical Methods in Natural Language Processing, pages 521-530. 